\title{
Infectious complications of induction treatment for acute myeloid leukaemia using the " $7+3$ " protocol without antibiotic prophylaxis -15 years of experience of one clinical site
}

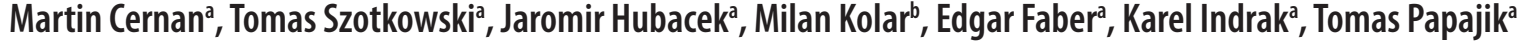

Background. Infectious complications during induction chemotherapy of acute myeloid leukaemia are very common. Prophylactic use of antibiotics however is an ongoing challenge in this situation due to bacterial multi-drug resistance. The aim of this study was to provide a comprehensive overview of the incidence of infectious complications in patients with AML undergoing induction therapy using the "7+3" protocol without routine antibiotic prophylaxis at one clinical site providing specialised haematological care in the Czech Republic, over a period of 15 years. The study also evaluates the aetiological spectrum of causative agents and the development of antibiotic resistance in the context of the use of the various classes of antibiotics. The analysis includes evaluation of the importance of risk factors for infectious complications and their impact on treatment of the underlying disease. The data are compared with published figures for similar cohorts of patients.

Patients and Methods. This study presents a retrospective analysis of infectious complications in 242 patients with acute myeloid leukaemia undergoing the first cycle of induction therapy without routine antibiotic prophylaxis in one clinical site in Czech Republic during years 2006-2020.

Results. A total of 363 febrile episodes (FE) were recorded. At least 1 FE during the induction was detected in 229 (94.6\%) patients. Clinically defined infection was the cause in 96 (26.4\%) FEs and blood stream infection in 69 (19.0\%) FEs. Both complications occurred simultaneously in 29 (8.0\%) FEs. 169 (46.6\%) FEs were evaluated as fever of unknown origin (FUO). The achievement of complete remission had a significant effect on the duration of the FE (6 vs. 9 days, $P=0.0005)$ and on the overall survival duration (79.3 vs. 6.5 months, $P<0.0001)$. Patients diagnosed with infection or FUO at diagnosis were significantly more likely to suffer from colonisation by multi-drug resistant bacterial strains at discharge ( $29.2 \%$ vs. $16.3 \%, P=0.022)$. This group of patients used antibiotic therapy for a significantly longer time (35 vs. 23 days, $P<0.0001)$. Infection was a contributing cause of death in $18(7.4 \%)$ patients. Mortality was significantly related to the failure to achieve complete remission $(P<0.0001)$.

Conclusion. Infectious mortality during induction treatment without routine antibiotic prophylaxis was comparable to the published cohorts with prophylaxis. Regular microbiology surveillance with adequate initial antibiotic treatment can compensate routine antibiotic prophylaxis with slower development of antibiotic resistance.

Key words: acute myeloid leukaemia, induction therapy, infectious complications, antibiotic prophylaxis, antibiotic resistance, mortality

Received: November 13, 2021; Revised: February 14, 2022; Accepted: February 15, 2022; Available online: March 1, 2022 https://doi.org/10.5507/bp.2022.010

(c) 2022 The Authors; https://creativecommons.org/licenses/by/4.0/

${ }^{a}$ Department of Haemato-Oncology, Faculty of Medicine and Dentistry, Palacky University Olomouc and University Hospital Olomouc, I.P.Pavlova 6, 77900 Olomouc, Czech Republic

${ }^{b}$ Department of Microbiology, Faculty of Medicine and Dentistry, Palacky University Olomouc and University Hospital Olomouc, I.P.Pavlova 6, 77900 Olomouc, Czech Republic

Corresponding author: Martin Cernan, e-mail:martin.cernan@fnol.cz

\section{INTRODUCTION}

Acute myeloid leukaemias (AML) constitute a heterogeneous group of haematopoietic malignant disorders. The curative treatment of AML is based on induction chemotherapy, with the aim of inducing complete remission (CR) of the disease. Infectious complications are a major cause of mortality during induction chemotherapy ${ }^{1}$. Lack of mature granulocytes caused by the underlying disease or chemotherapy is the most significant risk factor for infectious complications. The association between the decreasing number of circulating mature leukocytes and the rising incidence of infectious complications in patients with acute leukaemia was reported by Bodey et al. as early as 1966 (ref. $^{2}$ ). The risk of infection significantly increases with the extent of the decrease and the duration of neutropenia. Other risk factors for infectious complications include comorbidities, mucocutaneous barrier disruption due to invasive entry points or chemotherapy, and colonisation by virulent pathogens ${ }^{3}$. Febrile neutropenia is one of the most common emergencies in AML patients undergoing intensive cancer therapy. While empirical antibiotic treatment led to a significant reduction in mortality from the initial infection, infections caused by 
multi-drug resistant bacterial strains have soon become a new therapeutic challenge ${ }^{4}$. Despite the advances in supportive care, induction chemotherapy remains one of the most high-risk phases of AML therapy, with mortality at the level of $5 \%$ in developed countries, but up to $15-25 \%$ in developing countries. This is caused by the high incidence of infections by multi-drug resistant bacteria due to the unregulated use of antibiotics ${ }^{5-9}$.

The aim of this study was to provide a comprehensive overview of the incidence of infectious complications in patients with AML undergoing induction therapy using the " $7+3$ " protocol without routine antibiotic prophylaxis, at one clinal site providing specialised haematological care in the Czech Republic, over a period of 15 years. The study also evaluates the aetiological spectrum of causative agents and the development of antibiotic resistance in the context of the use of the various classes of antibiotics. Furthermore, the analysis includes evaluation of the importance of the risk factors of infectious complications, their influence on the results of the therapy of the underlying disease. The acquired data are compared with published data for similar cohorts of patients.

\section{PATIENTS AND METHODS}

\section{Patients and induction chemotherapy}

The study is a retrospective descriptive analysis of a group of patients with AML treated at the Department of Haemato-Oncology, Faculty of Medicine, Palacky University and the University Hospital Olomouc during the period from $1 / 1 / 2006$ to $31 / 12 / 2020$. The group included all adult patients ( $\geq 18$ years) with non-M3 AML according to ELN (European Leukemia Net) 2017 diagnostic criteria, who went through the first cycle of induction chemotherapy according to the " $7+3$ " protocol (cytosine arabinoside $100-200 \mathrm{mg} / \mathrm{m}^{2} /$ day in a continuous 24-hour infusion for 7 days, in combination with mitoxantrone $7.5-12 \mathrm{mg} / \mathrm{m}^{2}$ or daunorubicin $45-90 \mathrm{mg} / \mathrm{m}^{2}$ or idarubicin $12 \mathrm{mg} / \mathrm{m}^{2}$ in an hour-long infusion for a total of 3 days), or in combination with gemtuzumab ozogamicin and/or a FLT3 inhibitor ${ }^{10}$. During the period of interest, a total of 258 patients underwent the first cycle of induction chemotherapy. Out of this cohort, 242 patients received induction according to the " $7+3$ " protocol. 16 patients were treated based on other protocols and were not included in the analysis. Midostaurin was administered at a dose of $100 \mathrm{mg} /$ day in selected patients with a proven FLT3ITD or FLT3-TKD mutation on induction days 8-21. In one case, the therapy followed the AC 220-A-U302 study protocol (a randomised, double-blind, placebo-controlled phase III study with quizartinib in combination with induction chemotherapy) (ref. ${ }^{11}$ ). Gemtuzumab ozogamicin was administered at a dose of $3 \mathrm{mg} / \mathrm{m}^{2}$ (maximum $5 \mathrm{mg}$ per application) on induction days 1, 4 and 7 .

\section{Supportive therapy and management of infectious complications}

The patients lay in 2-bed rooms with their own bathrooms, equipped with air conditioning without HEPA filtration. All patients had a central venous access introduced at the start of the therapy: 234 (96.7\%) patients had a central venous catheter (predominantly cannulation of the internal jugular vein or subclavian vein) and 8 (3.3\%) patients had a PICC (peripherally inserted central catheter). A complete culture examination was performed in all patients upon admission (throat swab, urine, stool, possibly perianal swab, or swab from an infectious focus), with serological testing (chlamydia, mycoplasma, CMV, Toxoplasma gondii, infectious hepatitis, syphilis and HIV), including the PCR test for SARS_CoV_2, starting March 2020. Further culture examinations were performed at least once a week or in case of signs of infection requiring the initiation or changes to anti-infective therapy. Antibiotic prophylaxis was not routinely used in this cohort, with the exception of 2 patients (artificial heart valve, bleeding into the gastrointestinal tract). Antifungal prophylaxis was utilised in accordance with current guidelines and indication criteria for the individual antifungals. Antifungal prophylaxis was based on posaconazole (suspension or tablets) in 105 (43.4\%) patients, fluconazole in $97(40.1 \%)$ and itraconazole in 10 (4.1\%) patients. Other antifungals for primary antifungal prophylaxis - anidulafungin, micafungin and voriconazole - were used in less than 10 patients each. 20 (8.3\%) patients underwent the induction therapy without antifungal prophylaxis. Antiviral prophylaxis with aciclovir or pneumocystis pneumonia prophylaxis with co-trimoxazole was not routinely used.

Culture examinations were performed in case of fever or clinical signs of infection, including 2 pairs of blood cultures. Empirical first-line antibiotic therapy, usually amoxicillin/clavulanate and gentamicin or piperacillin/ tazobactam, was initiated, followed by adjustments based on the culture test results. If the fever states persisted or the clinical signs of infection progressed with no infectious agents detected in the culture, the treatment was escalated to the 2 nd line of empirical antibiotic therapy with ceftazidime/meropenem, vancomycin and amikacin/ gentamicin, followed by the 3rd line based on cefepime/ tigecycline and teikoplanin. No strict indications for antibiotic therapy discontinuation have been established; clinical signs of infection, dynamics of infection laboratory markers and expected duration of neutropenia were always considered. Antifungal prophylaxis/therapy was adjusted in case of clinical, imaging, or laboratory evidence of invasive fungal infection or persistence of fever unresponsive to broad-spectrum antibiotic therapy for 3-5 days. The granulocyte growth factors (G-CSF) were not used routinely to shorten the duration of neutropenia. These agents were indicated only in life-threatening infectious complications in monotherapy or in combination with granulocyte deposits, or in case of slow granulopoiesis regeneration after the induction treatment $(>30$ days after the initiation of therapy). Invasive fungal infections (IFIs) were classified according to the European Organization for Research and Treatment of Cancer and the Mycosis Study Group (EORTC/MSG) (ref. ${ }^{12}$ ). Antibiotic resistance was evaluated based on international recommendations published by Magiorakos et al. ${ }^{13}$. 


\section{Infectious complications evaluation methods}

A febrile episode (FE) was defined as an event where a patient with a fever $\geq 38.0{ }^{\circ} \mathrm{C}$ required the systemic initiation/change of antimicrobial therapy in the event of suspected infection based on clinical, laboratory, or imaging studies. FE end was defined as the day the body temperature was below $37.5^{\circ} \mathrm{C}$ for at least 3 consecutive days without antipyretic agents or corticosteroids. The FE aetiology was subsequently classified as a clinically defined infection according to the site affected - oral infections (odontogenic infections, stomatitis, gingivitis), ENT infections (sinusitis, pharyngitis, rhinitis), respiratory tract infections (pneumonia, bronchitis), soft tissue infections (phlegmon, folliculitis), gastrointestinal tract infections (clostridial enteritis, perianal abscess, colitis), urogenital infections (urosepsis, gynaecological infections) and others. The occurrence of herpes infections was evaluated regardless of the concurrent presence of FE. Bloodstream infection (BSI) was defined as a positive culture detection of a bacterial or fungal agent in blood during a febrile episode. The detection of common skin commensals was considered a bloodstream infection when detected in 2 specimens collected at different timepoints or specimens from different vessels, in combination with the presence of systemic signs of infection (fever, chills or hypotension). Catheter infection was defined via culture confirmation of blood culture agents and an extracted central venous cannula. Septic shock was considered to be a blood stream infection requiring vasopressors to maintain mean arterial pressure $\geq 65 \mathrm{mmHg}$. FEs without confirmed aetiology of infection based on clinical, microbiological or imaging examinations were classified as "fever of unknown origin" (FUO). If an infectious aetiology of fever was detected during FE initially classified as FUO, this episode was subsequently classified as a clinically or microbiologically defined infection.

\section{Other parameters evaluated}

Demographic data and characteristics of the underlying disease according to ELN 2017 criteria were further analysed in the patients in the cohort ${ }^{10}$. Furthermore, selected laboratory parameters were analysed when diagnosing AML - leukocyte count, the proportion of blasts in the peripheral blood and bone marrow, the absolute number of neutrophils, lymphocytes and the level of C-reactive protein. The duration of the anti-infective therapy and the use of the various groups of antibiotics, antifungals and antivirals were assessed as well. The effect on the occurrence of infectious complications during the induction chemotherapy was evaluated for selected parameters. The data of all patients acquired from available medical records were processed anonymously, in accordance with the internal regulations of the University Hospital Olomouc and the Declaration of Helsinki.

\section{Statistical analysis}

IBM SPSS Statistics version 23 (Armonk, NY: IBM Corp.) was used for data analysis. The Chi-square test or Fisher's exact test was used to compares the qualitative variables. The Kruskal-Wallis test with the Mann-Whitney post-hoc tests were used to compare the quantitative data. The Bonferroni significance correction was used for the multiple comparison (comparison of three observation periods). Data normality was verified using the ShapiroWilk test. The Kaplan-Meier analysis was used to compare OS (Overall survival). All tests were performed at a significance level of 0.05 .

\section{RESULTS}

\section{Demographic data and induction therapy}

The group consisted of a total of 242 patients - 124 men and 118 women with a median age of 57.0 years (20.9 - 72.3) and 55.4 years (24.4 - 69.8), respectively. $191(78.9 \%)$ cases of the disease were classified as de novo AML. A total of $51(21.1 \%)$ cases were classified as secondary AML: 30 (58.8\%) patients had a history of myelodysplastic syndrome (MDS) and 21 (41.2\%) had undergone anticancer therapy for other haematological malignancies or solid tumours. Normal karyotype was found in 114 (47.1\%) patients and karyotype abnormalities were detected in 128 (52.9\%) patients. Based on the ELN 2017 risk stratification, AML prognosis was considered favorable in 55 (22.7\%) patients, intermediate-risk in 106 $(43.8 \%)$ and high-risk in 81 (33.5\%) patients.

$141(58.3 \%)$ patients underwent induction chemotherapy in the " $7+3$ " protocol with daunorubicin and $90(37.2 \%)$ patients, with mitoxantrone. 11 (4,5\%) patients received induction therapy " $7+3$ " in a different composition based on other anthracyclines, FLT3 inhibitors and/or gemtuzumab ozogamicin. Induction therapy was completed in $226(93.4 \%)$ patients who initiated it. The median timepoint of premature discontinuation of induction due to complications was day 5 of therapy. Fever or sepsis was the cause of premature discontinuation in 9 (3.7\%) patients; pneumonia, in $6(2.5 \%)$ patients. There was $1(0.4 \%)$ case of haemorrhagic diathesis. Complete remission after the induction therapy was achieved in 139 (57.4\%) patients.

\section{Infectious complications before the initiation of the induction therapy}

Infection or FUO was detected in 144 (59.5\%) patients before the initiation of the induction therapy. FUO was present in $60(41.7 \%)$ patients, infections in $84(58.3 \%)$ of these patients. $26(31.0 \%)$ patients had a respiratory infection; the second most numerous group consisted of ENT infections - in 25 (29.8\%) patients. Infections in the oral cavity, including odontogenic foci, were reported in $18(21.4 \%)$ patients and soft tissue infections in $14(16.7 \%)$ patients. Gastrointestinal infection, gynaecological or urological infection were present in $2(2.4 \%)$ patients each. Sepsis caused by $S$. Enteritidis was reported in one case. $6(7.1 \%)$ patients had initially 2 infectious foci. There were $98(40.5 \%)$ patients without infectious complications before the initiation of induction. $146(60.3 \%)$ patients received systemic antibiotic therapy before the induction, including 2 patients in the prophylactic indication. 


\section{Infectious complications of the induction therapy}

363 febrile episodes were recorded in a group of 242 patients (median $1 \mathrm{FE} /$ induction therapy) during the first cycle of induction therapy. One FE was recorded in $119(49.2 \%)$ patients and 2 FEs in $88(36.4 \%)$ patients. 20 ( $8.3 \%)$ patients had 3 FEs and only $2(0.8 \%)$ patients had 4 FEs. Induction therapy without FE was recorded in 13 patients (5.4\%). Clinically defined infection as the cause of FE was found in $96(26.4 \%) \mathrm{FE}$ cases and BSI was the cause of $69(19.0 \%)$ FEs. A total of 29 (8.0\%) FEs were associated with a concomitant clinically defined infection and BSI. 169 (46.6\%) FEs were considered FUO.

Clinically defined infections (regardless of concomitant BSI) were documented in 125 (34.4\%) FEs. 136 clinically defined infections during induction were recorded in the study group. 11 patients had concomitant affection in 2 anatomical sites. The most common type was respiratory tract infections, especially pneumonia, accounting for $79(58.1 \%)$ of documented infections. The second most common group included infections in the oral cavity, representing $15(11.0 \%)$ cases, followed by gastrointestinal tract infections - 14 (10.3\%) cases of clinically defined infections. Clostridioides difficile infection (CDI) was detected in 7 patients. In this subgroup, it was the cause of FE in 3 cases and 4 other cases were not accompanied by fever. ENT infections were as common as soft tissue infections - 11 (8.1\%) cases. Urogenital infections were recorded in 3 (2.2\%) cases. Acute perimyocarditis, infectious endocarditis and splenic abscess were observed as additional infectious complications in one case each. Herpetic infections were recorded in 23 (9.5\%) patients in the cohort: orofacial involvement (herpes labialis) in 17 and perianal involvement in 3 patients. Shingles was recorded in 3 patients.

BSI was detected, regardless of the concurrent clinically defined infection, in a total of 98 (27.0\%) FEs. A Gram-positive $\left(\mathrm{G}^{+}\right)$agent was the cause of $53(54.1 \%)$ BSIs and a Gram-negative (G-) agent in 35 (35.7\%) BSIs. In $8(8.2 \%)$ cases, both $\mathrm{G}+$ and $\mathrm{G}$ - agents were detected. There were also $2(2.0 \%)$ cases of candidiasis. Concurrent 2 pathogens in blood culture were detected in 11 BSI cases and even 3 pathogens in 1 case, so a total of 109 bacterial strains and 2 candida species were detected during the period monitored.

Pseudomonas aeruginosa and Klebsiella pneumoniae, as the most common G- agents, were recorded in blood culture in 10 BSI cases each. 8 of the 10 strains of $K$. pneumoniae in blood culture were ESBL (extended spectrum beta-lactamases) positive. E. coli was detected in blood culture in 8 cases; only one strain was an ESBL producer. Enterobacter cloacae, Acinetobacter sp. and Stenotrophomonas maltophilia were recorded in 3 cases each. Both cases with Klebsiella variicola were ESBLpositive. Additional G- pathogens were recorded in isolated cases -6 isolates in total.

A representative of the genus Staphylococcus was detected in the blood culture in 47 BSI cases: most commonly S. epidermidis $23 / 47$ (48.9\%), followed by $S$. haemolyticus $12 / 47$ (25.5\%) and S. hominis 3/47 (6.4\%). There were two cases of $S$. capitis and $S$. aureus each (nei- ther strain was methicillin-resistant or MRSA). Other staphylococci were detected only in isolated cases. The second most numerous group was the genus Enterococcus - 4 cases of Enterococcus faecium and E. faecalis each. None of the strains was vancomycin-resistant. Strains of Corynebacterium sp. and Streptococcus sp. were detected in blood culture in 4 and 3 cases, respectively. There was one case of Micrococcus sp. and Bacillus sp. each. Furthermore, 2 cases of candidiasis were diagnosed with C. krusei and C. lusitaniae as the causal pathogens.

18 vascular infections in the cohort were complicated by septic shock; 9 (50.0\%) cases were caused by G-agents, $7(38.9 \%)$ cases, by $\mathrm{G}+$ agents. Both $\mathrm{G}+$ and $\mathrm{G}$ - pathogens were detected in $2(11.1 \%$ ) cases. K. pneumoniae ( 3 of the 4 strains were ESBL+) and $E$. coli (none of the 4 strains was ESBL-positive) were most frequently detected in the blood cultures of the G- agents, followed by $P$. aeruginosa (3 strains) and 1 case of $K$. oxytoca. Representatives of the genus Staphylococcus were identified 7 times; there were 2 cases of Streptococcus sp. and one case of Bacillus cereus and Corynebacterium jeikeium each. There were 3 cases of septic shock with 2 agents detected in the blood culture and one case with 3 agents in the blood culture at the same time. Hence, a total of 23 bacterial strains were detected.

Invasive fungal infections were found in a total of 80/363 (22.0\%) FEs. 70 cases of IFI were considered possible, 6 cases probable and 4 cases proven IFIs according to EORTC/MSG criteria ( 2 cases of candidiasis, 1 case of pneumocystis pneumonia and 1 case of aspergillus pneumonia with systemic dissemination).

\section{Anti-infective therapy}

The median duration of antibiotic therapy in the cohort was 30 days (0-96 days). A total of 227 (93.8\%) patients received a penicillin-line antibiotic or a second generation of cephalosporins during hospitalisation. 198 (81.8\%) patients were administered a glycopeptide type antibiotic (vancomycin or teicoplanin) while 182 (75.2\%) patients received an antibiotic in the aminoglycoside group (gentamicin or amikacin). Due to the epidemiological situation and the occurrence of ESBL+ bacteria, the previously widely used third and fourth generation cephalosporins $(77 / 242 ; 31.8 \%)$ were gradually replaced with carbapenem type antibiotics (mainly meropenem), which were used in a total of $170(70.2 \%)$ patients. Tigecycline, effective against most vancomycin-resistant enterococci, was used in $50(20.7 \%)$ patients in the cohort. In contrast, fluoroquinolones (mainly ciprofloxacin) were used only in $38(15.7 \%)$ patients. 17 (7.0\%) patients received clarithromycin in the treatment of atypical bacterial pneumonia. Each of other antibiotics - clindamycin, metronidazole, linezolid and colistin - was used in less than 10 patients in the cohort.

The median duration of antifungal therapy/prophylaxis in the cohort was 32 (0-114) days. Initial antifungal prophylaxis was changed or antifungal therapy was started in patients without primary prophylaxis in 131 (54.1\%) patients during the induction. The most common substitution was voriconazole, in $69(52.7 \%)$ patients, followed by 
the amphotericin-B lipid complex in 30 (22.9\%) patients. Itraconazole was used in further lines of therapy in 21 (16.0\%) patients, micafungin in $14(10.7 \%)$ and posaconazole in 12 (9.2\%) patients. Each of other antifungal drugs - fluconazole, anidulafungin or caspofungin - were used in less than 10 patients within modification of the initial antifungal prophylaxis/therapy. Up to 2 drugs were gradually changed in 24 patients and 3 antifungal drugs were used in 6 patients. $6 / 20(30.0 \%)$ patients who started induction therapy without antifungal prophylaxis required no antifungal therapy until the end of hospitalisation. 105 (43.4\%) patients completed induction therapy without initial antifungal prophylaxis changes.

Aciclovir antiviral therapy/prophylaxis was used in a total of $86(35.5 \%)$ patients in the cohort. The median duration of use was 19 (2-51) days. 8 patients with H1N1 pneumonia or a history of high-risk contact received oseltamivir as prophylaxis.

Granulopoiesis regeneration after chemotherapy was supported using granulocyte growth factor (G-CSF) in $50(20.7 \%)$ patients in the cohort and $7(2.9 \%)$ patients received granulocyte transfusions. Both treatment modalities were simultaneously used in 5 patients. The median duration of neutropenia (absolute neutrophil count $\leq 0.5 \mathrm{x}$ $10^{\wedge} 9 / \mathrm{L}$ ) in 190 patients who received no G-CSF or granulocyte transfusion was 24 days. The median duration of neutropenia was 27 days in patients who required G-CSF or granulocyte transfusion.

\section{Colonisation by multi-drug resistant bacterial strains}

$12(5.0 \%)$ patients were colonised with multi-drug resistant bacterial strains on admission to the hospital, i.e. prior to the induction therapy. ESBL+ enterobacteria were detected in 8 patients, vancomycin-resistant enterococci (VRE) in 2 patients, and ESBL-positive bacteria and VRE simultaneously in 2 patients. 7 of 12 patients had a history of previous hospitalisation in their medical records; 2 of 5 patients, without a history of previous hospitali- sation, received antibiotic therapy. 58 (24.0\%) patients were colonised with multi-drug resistant bacterial strains at the discharge. The most commonly detected G- agent was $K$. pneumoniae in 26 patients (25 ESBL+ strains and one XDR strain), followed by $P$. aeruginosa in 7 patients (all XDR strains) and Enterobacter sp. (ESBL+) in 6 patients. $K$. variicola (ESBL+ strains) and Stenotrophomonas maltophilia (PDR and XDR strains) were detected in 4 patients; 2 ESBL + E. coli strains and 1 case of Morganella morganii (ESBL+) and Burkholderia cepacia complex (XDR strain). 2 concurrent multi-drug resistant strains of G- bacteria were found in 2 patients. Vancomycinresistant strains of Enterococcus faecium were detected in 14 patients: 5 patients were co-colonized with VRE and multi-drug resistant G- bacteria. No patient was found to have methicillin-resistant $S$. aureus at the discharge. The colonisation of patients with multi-drug resistant bacteria varied during the study period. Patients were significantly more often colonised with ESBL+ strains and VRE during the last 5 years of the period of interest $(P=0.002$ and $P<0.0001$, respectively). Despite no changes in the FE count during the induction $(P=0.308)$, the duration of antibiotic therapy was significantly prolonged $(P=0.019)$. Tigecycline $(P<0.0001)$ and carbapenems $(P=0.001)$ were used significantly more frequently, while the use of generation 3 and higher cephalosporins was significantly less frequent $(P<0.0001)$ during the last 5 years of the study period due to the rising resistance to this group of antibiotics. Table 1 shows the development of colonisation of patients with multi-drug resistant strains of bacteria, selected parameters of hospitalisation and use of antibiotics in the observed period.

\section{Infection at the diagnosis as a prognostic factor}

Significantly longer duration of hospitalisation (36 vs. 33 days, $P<0.0001$ ), duration of antibiotic and antifungal therapy/prophylaxis ( 35 vs. 23 days, $P<0.0001$ and 35 vs. 28.5 days, $P<0.0001$ ), and more common colonisation

Table 1. Colonisation by multi-drug resistant strains of bacteria at the discharge and antibiotic use according to time periods: 2006-2010 / 2011-2015 / 2016-2020.

\begin{tabular}{lcccc}
\hline & $2006-2010$ & $2011-2015$ & $2016-2020$ & $P$ \\
\hline Number of patients & 83 & 85 & 74 & - \\
Colonisation by ESBL strains & $6(7.2 \%)^{\mathrm{a}}$ & $11(12.9 \%)$ & $20(27.0 \%)^{\mathrm{b}}$ & 0.002 \\
Colonisation by XDR or PDR G- strains & $6(7.2 \%)^{\mathrm{a}}$ & $4(4.7 \%)$ & $3(4.1 \%)$ & 1.000 \\
Colonisation by VRE strains & $1(1.2 \%)$ & $0(0 \%)$ & $13(17.6 \%)^{\mathrm{c}}$ & $<0.0001$ \\
Duration of ATB therapy (days) - median (min-max) & $27(0-73)$ & $31(12-63)$ & $31.5(9-96)$ & 0.019 \\
Number of FEs / patient (average) & $116 / 83(1.40)$ & $135 / 85(1.59)$ & $112 / 74(1.51)$ & 0.308 \\
FE duration (days) - median (min-max); average & $7(0-34) ; 8.7$ & $7(0-37) ; 9.4$ & $8(0-23) ; 8.6$ & 0.970 \\
Use of carbapenems (number of patients) & $46(55.4 \%)$ & $67(78.8 \%)$ & $57(77.0 \%)$ & 0.001 \\
Use of glycopeptides (number of patients) & $69(83.1 \%)$ & $72(84.7 \%)$ & $57(77.0 \%)$ & 0.424 \\
Use of tigecycline (number of patients) & $2(2.4 \%)$ & $22(25.9 \%)$ & $26(35.1 \%)$ & $<0.0001$ \\
Use of aminoglycosides (number of patients) & $57(68.7 \%)$ & $69(81.2 \%)$ & $56(75.7 \%)$ & 0.171 \\
Use of fluoroquinolones (number of patients) & $16(19.3 \%)$ & $12(14.1 \%)$ & $10(13.5 \%)$ & 0.541 \\
Use of third-generation and higher cephalosporins (number & $46(55.4 \%)$ & $23(27.1 \%)$ & $8(10.8 \%)$ & $<0.0001$ \\
of patients) & & & &
\end{tabular}

ESBL - extended-spectrum beta-lactamases, XDR - extensively drug resistant, PDR - pandrug resistant, G- gram negative, VRE - vancomycinresistant enterococci, ATB - antibiotics, FE - febrile episode, ${ }^{a}$ one patient with both ESBL+ and XDR strain, ${ }^{\mathrm{b}}$ one patient with 2 ESBL+ strains in the group, ${ }^{\mathrm{c}}$ five patients with ESBL+ and VRE in the group, 
with multi-drug resistant strains of bacteria at the discharge $(29.2 \%$ vs. $16.3 \%, P=0.002)$ were present in the group of 144 (59.5\%) patients diagnosed with infectious complications or FUO at the diagnosis. In this group, a non-significantly higher number of complete remissions ( 58.3 vs. $56.1 \%, P=0.733$ ) and a lower number of FEs during the induction ( 1.46 vs. $1.56, P=0.437$ ) were found. There were no significant differences in overall survival (OS) ( 14.4 vs. 19.1 months, $P=0.650$ ) or mortality during induction ( $9.7 \%$ vs. $6.1 \%, P=0.381$ ). This group of patients had also significantly higher leukocytosis, the proportion of blasts in the peripheral blood and bone marrow, and CRP $(P<0.0001)$ when diagnosed with AML compared to the group of patients without initial infectious complications or FUO. Table 2 shows a comparison of selected hospitalisation parameters and laboratory findings in the group of patients initially diagnosed with infection or FUO and in patients without infectious complications.

\section{Prognostic significance of achieving complete remission}

Patients who achieved complete remission of the underlying disease had non-significantly fewer FEs during the induction ( 1.42 vs. $1.62, P=0.153$ ), but these were significantly shorter ( 6 vs. 9 days, $P=0.0005$ ). The duration of neutropenia $\left(\leq 0.5 \times 10^{\wedge} 9 / \mathrm{L}\right)$ was significantly shorter $(24$ vs. 27 days, $P=0.006$ ) compared to the group of patients who did not reach CR. Patients who achieved complete remission were significantly more commonly colonised with resistant bacterial strains at the discharge (28.8\% vs, $17.6 \%, P=0.046)$. The overall survival in this group was significantly longer ( 79.3 vs. 6.5 months, $P<0.0001)$. Table 3 provides an overview of the effect of achieving complete remission on selected parameters of hospitalisation and antibiotic agent use in the patient cohort.

\section{Mortality}

A total of 20 patients in the study group died during the first cycle of the induction treatment. The cause of death was pneumonia in $14(70.0 \%)$ patients: of this, 2 cases had COVID-19 pneumonia with respiratory failure. Pneumonia was complicated by CNS bleeding in one case. Death was caused by multiorgan failure in 4 (20.0\%) patients and fatal CNS bleeding was recorded in $2(10.0 \%)$ patients. These were complications of therapyinduced cytopenia and/or persistence of the underlying disease after the treatment in all the cases. Mortality during induction was significantly related to the failure to achieve complete AML remission $(P<0.0001)$.

\section{DISCUSSION}

The study provides a comprehensive analysis of infectious complications in AML patients who underwent induction chemotherapy according to the " $7+3$ " protocol in one clinical site providing specialised haematological care in the Czech Republic, without routine antibiotic prophylaxis. Numerous papers have been published on the occurrence of infectious complications during the induction therapy of AML, but most of these studies are at least 10 years old. This study presents one of the most extensive cohorts of patients treated at one site ever published. However, comparison of the results is problematic due to the large heterogeneity of the study population (age, de novo vs. secondary AML, performance status), induction therapy type or the strategy of antibiotic, antifungal and antiviral prophylaxis matching the time the study was performed. Significant differences can be seen between the results from developed and developing countries ${ }^{7}$.

Table 2. Infection and FUO at the diagnosis as a prognostic factor.

\begin{tabular}{lccc}
\hline & Infection or FUO & No infection & $P$ \\
\hline Number of patients & 144 & $98^{c}$ & - \\
Age (years) - median (min-max) & $55.4(20.9-72.3)$ & $57.6(22.0-69.8)$ & 0.077 \\
Number of FEs / patient (average) & 1.46 & 1.56 & 0.437 \\
Duration of FE (days) - median (min-max) & $7(0-37)$ & $7(0-33)$ & 0.249 \\
Duration of hospitalisation (days) - median (min-max) & $36(13-73)$ & $33(19-117)$ & $<0.0001$ \\
Duration of ATB therapy (days) - median (min-max) & $35(12-73)$ & $23(0-96)$ & $<0.0001$ \\
Duration of AF therapy (days) - median (min-max) & $35(12-73)$ & $28.5(0-114)$ & $<0.0001$ \\
Time from admission to induction (days) - median (min-max) & $6(0-37)$ & $2.5(0-9)$ & $<0.0001$ \\
Colonisation by multidrug-resistant strains at discharge & $42(29.2 \%)$ & $16(16.3 \%)$ & 0.022 \\
Achieving of complete disease remission & $84(58.3 \%)$ & $55(56.1)$ & 0.733 \\
Death during induction & $14 \mathrm{~d}^{\mathrm{d}}(9.7 \%)$ & $6(6.1 \%)$ & 0.318 \\
Overall survival (months) - median (min-max) & $14.4(4.9-23.8)$ & $19.1(12.4-25.8)$ & 0.650 \\
Leukocytes (x10^9/L) - median (min-max) & $32.26(0.6-381.25)$ & $6.7(0.8-221.38)$ & $<0.0001$ \\
Blasts in MD (\%) - median (min-max) & $44(0-98)$ & $23(0-86)$ & $<0.0001$ \\
Blasts in BM (\%) - median (min-max) & $66.5(20-97)$ & $43(20-96)$ & $<0.0001$ \\
ANC below 0.5 x 10^9/L (number of patients) & $31(21.5 \%)$ & $35(35.7 \%)$ & 0.015 \\
Lymphocytes (x10^9/L) - median (min-max) & $3.13(0.3-89.9)$ & $2.3(0.48-48)$ & 0.067 \\
CRP (mg/l) - median (min-max) & $53.05(1-329.1)$ & $8(0.6-197.3)$ & $<0.0001$ \\
\hline
\end{tabular}

FUO - fever of unknown origin, ${ }^{a}$ ESBL, XDR, PDR or VRE, ${ }^{\mathrm{b}}-95 \% \mathrm{CI}$ (confidence interval), MD - manual differential, BM - bone marrow, ANC - absolute neutrophil count, CRP - C-reactive protein, FE - febrile episode, ATB - antibiotic agent, AF - antifungal agent, ${ }^{c} 2$ patients had prophylactic ATB (artificial valve, GI tract bleeding), ${ }^{\mathrm{d}} 2 \mathrm{x}$ bleeding into the CNS, 
The demographic characteristics of our cohort were not affected by the selection of patients based on age or by strict inclusion of only de novo AML; all patients who were able to undergo induction therapy according to the " $7+3$ " protocol were included in the study. Compared to published preselected groups of patients, the median age in our group was higher, patients with secondary AML accounted for a total of $21.1 \%$ of the cohort and up to $33.5 \%$ of patients had a high-risk disease according to the ELN 2017 stratification. For example, Cannas et al. reported $89 \%$ of CRs in a cohort of 459 patients aged 17-50 years (median age 46 years) with de novo AML undergoing induction therapy ${ }^{14} .231$ (95.5\%) patients in our group underwent induction therapy based on the " $7+3$ " protocol consisting of cytosine-arabinoside + anthracycline due to the retrospective nature of this analysis, which included patients treated since 2006. Modern drugs (gemtuzumab ozogamicin, FLT-3 inhibitors) were only registered in the Czech Republic in the last years of the study period. The number of complete remissions achieved $(57.4 \%)$ is therefore lower in comparison with recent works reporting on preselected groups of patients with a CR rate exceeding $70 \%$ (ref. ${ }^{15,16}$ ). Failure to achieve complete remission was significantly associated negatively with higher mortality in our cohort (predominantly due to infectious complications) during the induction and with overall survival $(P<0.0001)$. The characteristics of the cohort and the therapy used, affecting the probability of achieving CR, are thus important variables in the assessment of the incidence of infectious complications and mortality in patients undergoing AML induction treatment.

A total of $84(34.7 \%)$ patients had a clinically defined infection and $60(24.8 \%)$ patients had FUO before initiating the induction therapy. Respiratory tract infections, mainly pneumonia, were recorded most frequently.
Pandian et al. reported initial clinically defined infection in $31.0 \%$ and FUO in $10.4 \%$ of patients. Induction mortality was significantly higher in patients with an infection at diagnosis ( $28 \%$ vs. $7 \%, P=0.01)$ and a higher total leukocyte count $(P=0.02)$ (ref. $\left.{ }^{7}\right)$. The effect of infection or FUO at AML diagnosis on mortality was not statistically significant in our cohort $(9.7 \%$ vs. $6.1 \%, P=0.318)$; this group of patients had also significantly higher leukocyte counts, higher proportion of blasts in peripheral blood and bone marrow and significantly more common neutropenia $\left(\leq 0.5 \times 10^{\wedge} 9 / \mathrm{L}\right)$, which is a risk factor for infection. Buckley et al. reported that neutropenia below 0.5 $\mathrm{x} 10^{9} / \mathrm{L}$ at the diagnosis was significantly associated with the development of fever, infection, and bacteremia during the induction therapy ${ }^{17}$. Paraneoplastic aetiology of fever in some patients initially presenting with FUO cannot be ruled out. It is often indistinguishable from emergent febrile neutropenia in neutropenic patients, which requires initiation of antibiotic therapy ${ }^{18}$.

Infectious complications were very common during the induction therapy. Only $13(5.4 \%)$ patients had no infectious complication requiring systemic use of antibiotic therapy. Lech-Maranda et al. published their findings on a cohort where $72 \%$ patients received antibiotic prophylaxis during the AML induction therapy. Infectious complications were reported in $282(91.3 \%)$ patients. A total of 443 febrile episodes were observed in a cohort of 309 patients (1.43 FE / induction) (ref. ${ }^{19}$ ). In our cohort, the average number of FEs during induction was 1.5 (363 FEs / 242 patients). A non-significantly lower number of FEs was recorded in patients with an infection or FUO at the diagnosis and who underwent antibiotic therapy with possibly the same effect as primary antibiotic prophylaxis at the start of the induction treatment (1.46 vs. $1.56, P=0.437)$. In the group of clinically defined infec-

Table 3. Effect of achieving complete remission on selected parameters of hospitalisation and use of antibiotics in the patient cohort.

\begin{tabular}{lccc}
\hline & & & \\
& CR achieved & CR not achieved & $P$ \\
\hline Number of patients & 139 & 102 & - \\
Age (years) - median (min-max) & $55.3(20.9-69.9)$ & $57.7(22.0-72.3)$ & 0.078 \\
Number of FEs / patient (average) & $197 / 139(1.42)$ & $165 / 102(1.62)$ & 0.153 \\
ANC duration below 0.5 x 10^9/1 (days) - median (min-max) & $24(1-46)$ & $27(1-53)$ & 0.006 \\
Duration of ATB therapy (days) - median (min-max) & $29(0-73)$ & $30.5(10-96)$ & 0.143 \\
Duration of AF therapy (days) - median (min-max) & $32(0-73)$ & $33(10-114)$ & 0.345 \\
Duration of FE (days) - median (min-max) & $6(0-37)$ & $9(0-37)$ & 0.0005 \\
Death during induction & $0(0 \%)$ & $20(19.6 \%)$ & $<0.0001$ \\
Infection/FUO before induction - number of patients & $84(60.4 \%)$ & $60(58.8 \%)$ & 0.801 \\
Colonisation by multidrug resistant strains at discharge - number of patients & $40(28.8 \%)$ & $18(17.6 \%)$ & 0.046 \\
Carbapenems during induction - number of patients & $94(67.6 \%)$ & $76(74.5 \%)$ & 0.247 \\
Aminoglycosides during induction - number of patients & $107(77.0 \%)$ & $75(73.5 \%)$ & 0.538 \\
Glycopeptides during induction - number of patients & $107(77.0 \%)$ & $91(89.2 \%)$ & 0.014 \\
Tigecycline during induction - number of patients & $29(20.9 \%)$ & $21(20.6 \%)$ & 0.959 \\
Fluoroquinolones during induction - number of patients & $17(12.2 \%)$ & $21(20.6 \%)$ & 0.079 \\
Overall survival (months) - median (min-max) (95\% CI) & $79.3(26.7-131.8)$ & $6.5(3.5-9.5)$ & $<0.0001$ \\
\hline
\end{tabular}

The outcome of induction is not available in one patient. FE - febrile episode, ANC - absolute neutrophil count, ATB - antibiotic agents, AF antifungal agents, FUO - fever of unknown origin, ${ }^{a}$ ESBL, XDR, PDR or VRE, CI - confidence interval 
tions, the most common cases were lower respiratory tract infections, predominantly pneumonia, representing $31.0 \%$ of all clinically defined infections at diagnosis and $58.1 \%$ of infections during induction. The high incidence of pneumonia in patients undergoing induction therapy for AML has also been reported by Barreda Garcia et al. The cumulative 28-day incidence of pneumonia in the cohort was $21.6 \%$ and was significantly related to mortality $(P<0.001)\left(\right.$ ref. $\left.^{20}\right) .14 / 20(70 \%)$ patients who died in our cohort had pneumonia. However, none of them were in complete remission of the disease.

74 episodes of bacteremia in AML patients undergoing induction therapy were reported in a cohort of PALG (Polish Adult Leukemia Group). G+ cocci were the aetiological agent in $47 \%$ and G- rods in $23 \%$ of bacteremia cases. Multiple agents were detected in the same number of episodes. Fungaemia was found in 7\% of episodes, always caused by Candida species. Septic shock was reported in 14/74 (18.9\%) bacteremia cases ${ }^{19}$. 18 of 98 (18.4\%) BSIs were complicated by septic shock in our cohort. Coagulase-negative Staphyloccoci and G- rods from Enterobacteriacea family (especially $E$. coli and $K$. pneumoniae) and $P$. aeruginosa strains were the most common causative $\mathrm{G}+$ and $\mathrm{G}-$ agents, as in other published study groups. However, antibiotic prophylaxis of fluoroquinolones with a dominant effect against G- agents of BSI may also contribute to the ratio of $\mathrm{G}^{+}$and $\mathrm{G}-$ agents in the results ${ }^{19,21-23}$.

Primary antifungal prophylaxis was used in $91.7 \%$ of patients. The choice of the antifungal agent was affected by product availability during the period followed. The second-generation azoles, mainly posaconazole, have been used predominantly since 2014. The role of IFI in infectious complications cannot be ruled out in 80 FEs, although the number of probable and proven IFIs was low, only $10 / 242$ (4.1\%) of all patients. This result may be affected by the immediate availability of HRCT (High Resolution CT) of the lungs, which was always performed in case of suspected lung involvement or persistent fever even in broad-spectrum antibiotic therapy covering available culture outcomes. The availability of bronchoalveolar lavage, as well as the patients' willingness to undergo this examination, which would enable sampling for culture examination, was very low, similarly to the benefit of galactomannan testing. A change in pri- mary antifungal prophylaxis, or the initiation of antifungal therapy, was indicated in $54.1 \%$ of patients treated early preemptively with antifungals effective against filamentous fungi. Probable and proven IFIs were reported in a total of 6.2 - 15.9\% AML patients undergoing induction therapy. However, there were differences in primary antifungal prophylaxis in the cohorts ${ }^{14,19,22}$. A decrease in the incidence of IFI caused by the genus Aspergillus sp. has been reported in connection with the implementation of posaconazole in primary prophylaxis ( ref. $^{24}$ ).

An important observation in our group was a significant increase in the number of patients colonised with ESBL+ strains and VRE during the last 5 years of the period followed, requiring a substantially more frequent use of effective antibiotics - tigecycline and carbapenems, and conversely a decrease in the use of third-generation cephalosporins. An increase in VRE colonization in patients undergoing induction therapy for AML and MDS has also been described by Heisel et al. In the group of 235 patients, colonization was found in $2.5 \%$ of patients at admission, but in up to $59.0 \%$ of patients at the discharge. The use of intravenous vancomycin was a significant risk factor for colonization $(P=0.024)$ (ref. $\left.{ }^{25}\right)$. Using pulsed-field gel electrophoresis, Hricová et al. demonstrated clonality in $67 \%$ and a unique restriction profile in $33 \%$ of detected VRE strains in haemato-oncological patients treated at our site. All the strains detected were sensitive to linezolid and $96 \%$ strains were sensitive to tigecycline $^{26}$. Ballo et al. were unable to demonstrate an effect of MDRO (multi-drug resistant microorganisms) colonisation on overall survival in patients undergoing induction therapy of AML, with the exception of with carbapenem-resistant enterobacteria colonisation, which resulted in significantly higher mortality. The most common cause of death was sepsis, followed by progression of the underlying disease ${ }^{27}$. Regular microbiological screening is essential for the initiation of adequate initial antibiotic therapy in cases of febrile neutropenia in high-risk patients with hematologic malignancies. Our experience shows that routine primary antibiotic prophylaxis cannot replace information on the local epidemiological situation and detailed knowledge of the bacterial microflora in a particular patient. Infection caused by MDRO may be the cause of fever unresponsive to antibiotic therapy ${ }^{27}$.

Mortality in the PALG cohort with antibiotic prophy-

Table 4. An overview of infectious mortality in selected published cohorts.

\begin{tabular}{|c|c|c|c|c|c|c|}
\hline Cohort & $\begin{array}{l}\text { Number } \\
\text { of patients }\end{array}$ & $\begin{array}{l}\text { Age - median } \\
(\text { min-max) years }\end{array}$ & $\begin{array}{l}\text { Antibiotic } \\
\text { prophylaxis }\end{array}$ & $\begin{array}{c}\text { Infectious } \\
\text { mortality } \\
\mathrm{n}(\%)\end{array}$ & $P$ & Ref. \\
\hline Čerňan et al. & 242 & $56(21-72)$ & No & $18(7.4)$ & - & \\
\hline Syrjälä et al. & 327 & $48(16-66)$ & No & $12(3.7)$ & 0.234 & 21 \\
\hline Malagola et al. & 224 & $46(18-64)$ & Yes & $8(3.6)$ & 0.346 & 23 \\
\hline Lech-Maranda et al. & 309 & $45(16-60)$ & Yes & $31(10.0)$ & 1.000 & 19 \\
\hline De Rosa et al. & 81 & $50(23-69)$ & Yes & NS & - & 22 \\
\hline Cannas et al. & 459 & $46(17-50)$ & Yes & $7(1.5)$ & 0.0003 & 14 \\
\hline Kato et al. & 980 & $47(34-55)$ & Yes & $12(1.2)$ & $<0.0001$ & 28 \\
\hline
\end{tabular}

NS - not stated, - not applicable 
laxis was $36 / 309$ (11.7\%) patients, while 31 (10.0\%) patients died of infectious complications ${ }^{19}$. Malagola et al. reported death in 10/224 (4.5\%) patients during induction therapy of AML. Patients received antibiotic prophylaxis with levofloxacin. 8/10 patients died of an infection ${ }^{23}$. A total of 20 patients died in our group, of whom 18/242 (7.4\%) died of infectious complications. So, mortality was comparable to these cohorts where the patients received primary antibiotic prophylaxis $(P=1.000$ and $P=0.346$, respectively). However, other investigators using antibiotic prophylaxis reported significantly lower infectious mortality during AML induction (ref. ${ }^{14,28}$ ). A meta-analysis of 862 patients with acute leukemia undergoing intensive therapy, 356 of whom received antibiotic prophylaxis with levofloxacin, showed a significantly lower incidence of febrile neutropenia episodes, microbiologically documented infections and bacteremia $(P<0.00001)$. However, the use of antibiotic prophylaxis did not reduce mortality $(P=0.26)\left(\right.$ ref. $\left.^{29}\right)$. Table 4 shows an overview of infectious mortality in selected published cohorts.

Limitations of the present study include its retrospective nature and the long period of monitoring, associated with changes in the diagnostic and therapeutic guidelines, availability of anti-infective drugs and the examination methods, as well as the experience of the staff over the years. On the other hand, this study could capture the trends in the development of antibiotic resistance and the subsequent use of individual groups of antibiotics over a 15-year period. The aim of the study was to show the experience of one centre providing specialised haematological care with the administration of induction therapy in AML patients without antibiotic prophylaxis.

\section{CONCLUSION}

Infectious complications are the second most common cause of death after refractory disease in patients with hematologic malignancies. Here we describe the largest non-selected cohort of acute myeloid leukaemia patients undergoing induction treatment at a single institution without routine use of antibiotic prophylaxis. We can finally conclude that induction chemotherapy without routine antibiotic prophylaxis is not associated with any increased infectious mortality which was comparable to the cohorts with antibiotic prophylaxis reported in the literature. Mortality was significantly associated with failure to achieve complete remission of the underlying disease which is known to be strongly dependent on cytogenetic abnormalities and treatment course. These variables must be considered, when comparing mortality in various study groups. The occurrence of multi-drug resistant strains of bacteria tends to grow, despite regulated use of antibiotics. Our experience shows that precise information on the local epidemiological situation and detailed knowledge of the bacterial microflora in a particular patient are essential preconditions for the effective initial antibiotic therapy and cannot be replaced by routine antibiotic prophylaxis.
Acknowledgements: This work was supported by: SUG 87-14, MH CZ - DRO (FNOL, 00098892) and IGA_ LF_2021_001.

Author contributions: MC: manuscript writing, data collection and analysis, treatment of patients; TS, JH, EF, KI, TP: treatment of patients, critical revision of the manuscript; MK: microbiological diagnostics, critical revision of the manuscript. All authors agree with the final version of the manuscript.

Conflict of interest statement: None declared.

\section{REFERENCES}

1. De Kouchkovsky I, Abdul-Hay M. Acute myeloid leukemia: a comprehensive review and 2016 update. Blood Cancer Journal 2016;6:e441.

2. Bodey GP, Buckley M, Sathe YS, Freireich EJ. Quantitative relationships between circulating leukocytes and infection in patients with acute leukemia. Ann Intern Med 1966;64(2):328-40.

3. Giamarellou H, Antoniadou A. Infectious complications of febrile leukopenia. Infect Dis Clin North Am 2001;15(2):457-82.

4. Bent-Are Hansen $B A$, Wendelbo $\varnothing$, Bruserud $\varnothing$, Hemsing $A L$, Mosevoll KA, Reikvam H. Febrile Neutropenia in Acute Leukemia. Epidemiology, Etiology, Pathophysiology and Treatment. Mediterr J Hematol Infect Dis 2020;12(1):e2020009.

5. Othus M, Kantarjian H, Petersdorf S, Ravandi F, Godwin J, Cortes J, Pierce S, Erba H, Faderl S, Appelbaum FR, Estey E. Declining Rates of Treatment-Related Mortality in Patients with Newly-diagnosed AML Given "Intense" Induction Regimens: A Report from SWOG and MD Anderson. Leukemia 2014;28(2):289-92.

6. Philip C, George B, Ganapule A, Korula A, Jain P, Alex AA, Lakshmi KM, Sitaram, Abubacker FN, Abraham A, Viswabandya A, Srivastava VM, Srivastava A, Balasubramanian P, Mathews W. Acute myeloid leukaemia: challenges and real world data from India. Br J Haematol 2015;170(1):110-17.

7. Pandian J, Raghavan V, Manuprasad A, Shenoy PK, Nair CK. Infection at diagnosis-a unique challenge in acute myeloid leukemia treatment in developing world. Support Care Cancer 2020;28(11):544954.

8. Kayal S, Sengar M, Jain H, Bonda A, George B, Kulkarni UP, Balasubramanian P, Bhurani D, Ahmed R, Biswajit D, Jayachandran PK, Batra A, Kumar S, Mishra K, Bhattacharyya J, Philip CC, Bhattacharyya M, Dasappa L, Babu KG, Saldanha SC, Sharma A, Lakshmanan J, Durairaj J, Rudravaram VV, Mathews W, Kapoor R. Induction Related Mortality in Acute Myeloid Leukemia: Multivariate Model of Predictive Score from the Indian Acute Leukemia Research Database (INwARD) of the Hematology Cancer Consortium (HCC). Blood (2019);134 (S1):2615.

9. Van Boeckel TP, Gandra S, Ashok A, Caudron Q, Grenfell BT, Levin SA, Laxminarayan R. Global antibiotic consumption 2000 to 2010: an analysis of national pharmaceutical sales data. Lancet Infect Dis 2014;14(8):742-50.

10. Döhner H, Estey E, Grimwade D, Amadori S, Appelbaum FR, Büchner T, Dombret H, Ebert BL, Fenaux P, Larson RA, Levine LR, Lo-Coco F, Naoe T, Niederwieser D, Ossenkoppele GJ, Sanz M, Sierra J, Tallman MS, Tien HF, Wei AH, Löwenberg B, Bloomfield CD. Diagnosis and management of AML in adults: 2017 ELN recommendations from an international expert panel. Blood (2017);129(4):424-47.

11. Garcia-Horton A, Yee KW. Quizartinib for the treatment of acute myeloid leukemia. Expert Opin Pharmacother 2020;21(17):2077-90.

12. Donnelly JP, Chen CC, Kauffman CA, Steinbach WJ, Baddley JW, Verweij PE, Clancy CJ, Wingard JR, Lockhart SR, Groll AH, Sorrell TC, Bassetti M, Akan H, Alexander BD, Andes D, Azoulay E, Bialek R, Bradsher RW, Bretagne S, Calandra T, Caliendo AM, Castagnola E, Cruciani M, Cuenca-Estrella M, Decker CF, Desai SR, Fisher $B$, Harrison T, Heussel CP, Jensen HE, Kibbler CC, Kontoyiannis PD, Kullberg BJ, Lagrou K, Lamoth F, Lehrnbecher T, Loeffler K, Lortholary O, Maertens K, Marchetti O, Marr KA, Masur K, Meis JF, Morrisey CO, Nucci M, Ostrosky-Zeichner L, Pagano L, Patterson TF, Perfect JR, Racil Z, Roilides E, Ruhnke M, Prokop CS, Shoham S, Slavin MA, Stevens DA, Thompson GR, Vazquez JA, Viscoli C, Walsh 
TJ, Warris A, Wheat LJ, White PL, Zaoutis TE, Pappas PG. Revision and Update of the Consensus Definitions of Invasive Fungal Disease From the European Organization for Research and Treatment of Cancer and the Mycoses Study Group Education and Research Consortium. Clin Infect Dis 2020;71(6):1367-76.

13. Magiorakos AP, Srinivasan A, Carey RB, Carmeli Y, Falaga ME, Giske CG, Harbarth S, Hindler JF, Kahlmeter G, Olsson-Liljequist B, Paterson DL, Rice LB, Stelling J, Struelens MJ, Vatopoulos A, Weber JT, Monnet DL. Multidrug-resistant, extensively drug-resistant and pandrug resistant bacteria: an international expert proposal for interim standard definitions for acquired resistence. Clin Microbiol Infect 2012;18:268-81.

14. Cannas G, Pautas C, Raffoux E, Quesnel B, De Botton S, De Revel T, Reman O, Gardin C, Elhamri M, Boissel N, Fenaux P, Michallet $\mathrm{M}$, Castaigne S, Dombret $\mathrm{H}$, Thomas X. Infectious complications in adult acute myeloid leukemia: analysis of the Acute Leukemia French Association-9802 prospective multicenter clinical trial. Leuk Lymphoma 2012;53(6):1068-76.

15. Lambert J, Pautas $C$, Terré $C h$, Raffoux E, Turlure $P$, Caillot $D$, Legrand O, Thomas X, Gardin C, Gogat-Marchant K, Rubin SD, Benner RJ, Bousset $\mathrm{P}$, Preudhomme C, Chevret C, Dombret H, Castaigne S. Gemtuzumab ozogamicin for de novo acute myeloid leukemia: final efficacy and safety updates from the open-label, phase III ALFA-0701 trial. Haematologica 2019;104(1):113-19.

16. Aldoss I, Pullarkat V, Stein AS. Venetoclax-containing regimens in acute myeloid leukemia. Ther Adv Hematol 2021;12:2040620720986646.

17. Buckley SA, Othus M, Vainstein V, Abkowitz JL, Estey EH, Walter RB. Prediction of adverse events during intensive induction chemotherapy for acute myeloid leukemia or high-grade myelodysplastic syndromes. Am J Hematol 2014;89(4):423-28.

18. Pagano L, Caira M, Nosari A, Rossi G, Viale P, Aversa F, Tumbarello M, Hema e-Chart Group, Italy. Etiology of febrile episodes in patients with acute myeloid leukemia: results from the Hema e-Chart Registry. Arch Intern Med 2011;171(16):1502-03.

19. Lech-Maranda E , Seweryn M, Giebel S, Holowiecki J, PiatkowskaJakubas B, Piatkowska-Jakubas B, Wegrzyn J, Skotnicki A, Kielbinski M, Kuliczkowski K, Paluszewska M, Jedrzejczak WW, Dutka M, Hellmann A, Flont M, Zdziarska B, Palynyczko G, Konopka L, Szpila T, Gawronski K, Sulek K, Sokolowski J, Kloczko J, Warzocha K, Robak T. Infectious complications in patients with acute myeloid leukemia treated according to the protocol with daunorubicin and cytarabine with or without addition of cladribine. A multicenter study by the Polish Adult Leukemia Group (PALG). Int J Infect Dis 2010;14(2):132 40.

20. Garcia JB, Lei X, Wierda W, Cortes JE, Dickey BF, Evans SE, Ost DE. Pneumonia during remission induction chemotherapy in patients with acute leukemia. Ann Am Thorac Soc 2013;10(5):432-40.

21. Syrjälä $H$, Ohtonen $P$, Kinnunen $U$, Räty $R$, Elonen $E$, Nousiainen T, Jantunen E, Remes K, Itälä-Remes M, Silvennoinen R, Koistinen
P, Finnish Leukemia Group. Blood stream infections during chemotherapy-induced neutropenia in adult patients with acute myeloid leukemia: treatment cycle matters. Eur J Clin Microbiol Infect Dis 2010;29(10):1211-18.

22. De Rosa FG, Motta I, Audisio E, Frairia Ch., Busca A, Di Perri G, Marmont F. Epidemiology of bloodstream infections in patients with acute myeloid leukemia undergoing levofloxacin prophylaxis. BMC Infect Dis 2013;13:563.

23. Malagola M, Peli A, Damiani D, Candoni A, Tiribelli M, Martinelli G, Piccaluga PP, Paolini S, De Rosa F, Lauria F, Bocchia M, Gobbi M, Pierri I, Zaccaria A, Zuffa E, Mazza P, Priccolo G, Gugliotta L, Bonini A, Visani G, Skert C, Bergonzi C, Roccaro AM, Fili C, Fanin R, Baccarani $M$, Russo $D$. Incidence of bacterial and fungal infections in newly diagnosed acute myeloid leukaemia patients younger than $65 \mathrm{yr}$ treated with induction regimens including fludarabine: retrospective analysis of 224 cases. Eur J Haematol 2008;81(5):354-63.

24. Weinbergerová $B$, Kabut $T$, Kocmanová I, Drgoňa L, Kouba M Hričinová M, Gabzdilová J, Guman T, Petečuková V, Novák J, Forsterová K, Haber J, Žiaková B, Bojtárová E, Zavřelová A, Karas M, Chrenková V, Sedláček P, Tkáčiková B, Múdry P, Mallátová N, Timr P, Kolenová A, Tanušková D, Horáková J, Navrátil M, Chudej J, Sokol J, Rolencová M, Žák P, Cetkovský P, Mayer J, Ráčil Z. Změny v epidemiologii invazivních mykotických infekcí v českých a slovenských hematoonkologických centrech v letech 2005-2017: analýza dat FIND. Transfuze Hematol. Dnes 2019;2:186-96. [article in Czech]

25. Heisel RW, Sutton RR, Mascara GP, Winger GD, Weber DR, Lim $\mathrm{SH}$, Oleksiuk LM . Vancomycin-resistant enterococci in acute myeloid leukemia and myelodysplastic syndrome patients undergoing induction chemotherapy with idarubicin and cytarabine. Leuk Lymphoma 2017;58(11):2565-72.

26. Hricová K, Štosová T, Kučová P, Fišerová K, Bardoň J, Kolář M. Analysis of Vancomycin-Resistant Enterococci in Hemato-Oncological Patients. Antibiotics (Basel) 2020; 9(11):785.

27. Ballo O, Tarazzit I, Stratmann J, Reinheimer C, Hogardt $M$ Wichelhaus TA, Kempf V, Serve H, Finkelmeier F, Brandts C. Colonization with multidrug resistant organisms determines the clinical course of patients with acute myeloid leukemia undergoing intensive induction chemotherapy. PLoS One 2019;14(1):e0210991.

28. Kato H, Fujita H, Akiyama N, Kimura Sl, Hiramoto N, Hosono $\mathrm{N}$, Takahashi T, Shigeno K, Minamiguchi H, Miyatake J, Handa H, Kanda Y, Yoshida M, Miyawaki S, Ohtake S, Naoe T, Kiyoi $\mathrm{H}$, Matsumura I, Miyazaki Y. Infectious complications in adults undergoing intensive chemotherapy for acute myeloid leukemia in 2001-2005 using the Japan Adult Leukemia Study Group AML201 protocols. Support Care Cancer 2018;26(12):4187-98.

29. Owattanapanich W, Chayakulkeeree M. Efficacy of levofloxacin as an antibacterial prophylaxis for acute leukemia patients receiving intensive chemotherapy: a systematic review and meta-analysis. Hematology 2019;24(1):362-68. 